1 Short report

\title{
2 Chimpanzees communicate to coordinate a cultural practice
}

3 Zoë Goldsborough ${ }^{1,2 \dagger}$, Anne Marijke Schel ${ }^{1}$, Edwin J. C. van Leeuwen ${ }^{3,4 \dagger *}$

4

$5{ }^{1}$ Animal Ecology, Department of Biology, Utrecht University, Padualaan 8, 3584 CA

6 Utrecht, The Netherlands (ZG: zgoldsborough@outlook.com; AMS: A.M.Schel@uu.nl)

$7 \quad{ }^{2}$ Department for the Ecology of Animal Societies, Max Planck of Animal Behavior,

8 Bücklestraße 5a, 78467, Konstanz, Germany

$9 \quad{ }^{3}$ Behavioral Ecology and Ecophysiology Group, Department of Biology, University of

10 Antwerp, Universiteitsplein 1, 2610 Wilrijk, Belgium

$11{ }^{4}$ Centre for Research and Conservation, Royal Zoological Society of Antwerp, K. Astridplein

12 26, B 2018 Antwerp, Belgium

13

$14 \dagger$ contributed equally

15

16 *Corresponding author: ejcvanleeuwen@gmail.com, https://orcid.org/0000-0002-7729-2182

17

18 Key words: grooming handclasp, Pan troglodytes, communication, culture, coordination 


\section{Abstract}

20 Human culture is considered to differ from animal culture due to its interactive nature built

21 on shared intentionality and cognitive flexibility. Here, we investigated whether chimpanzees

22 use communication to engage in cultural practices by analyzing grooming handclasp (GHC)

23 interactions - a socio-cultural behavior requiring coordination. Previous accounts attributed

24 GHC initiations to behavioral shaping whereby the initiator physically molds the partner's

25 arm into the GHC posture. Using frame-by-frame analysis and matched-control methodology,

26 we find that chimpanzees use gestural communication to initiate GHC, which requires an

27 active and synchronized response from the partner. This showcases a behavioral expression

28 of joint commitment to engage in this shared cultural practice. Moreover, we show that GHC

29 initiators used various initiation strategies, attesting to situation-contingent interactional

30 flexibility. We conclude that chimpanzees can be jointly committed to a cultural practice,

31 which suggests that culture predicated on shared intentionality and flexible communication

32 may not be unique to the human species. 


\section{Introduction}

34 Culture - the inheritance of behavioral phenotypes through social learning (Laland and Janik,

35 2006) - lies at the heart of the biological success of the human species (Henrich, 2016). It is

36 thought to qualitatively differ from culture in non-human animals (henceforth 'animals') by

37 its communicative nature reflecting both shared intentionality and cognitive flexibility

38 (Enfield and Levinson, 2006; Tomasello, 2019). Shared intentionality is present in

39 collaborative interactions in which participants share psychological states with one another

40 (Tomasello and Carpenter, 2007). It facilitates mutual engagement and commitment from the

41 collaborators by synchronizing their attention and attuning their actions into adequate and

42 complementary action-response sequences. In human culture, two individuals may thus enter

43 a state of shared commitment to engage in a cultural ritual together (e.g., shaking hands when

44 meeting each other (Oxlund, 2020)), which allows for the coordination of the required actions

45 to pursue and complete the shared ritual (Bratman 1992; Enfield and Levinson 2006).

46 According to an influential view in developmental psychology, animals lack the

47 prerequisites for shared intentionality (Tomasello, 2019; Tomasello et al., 2012; Tomasello

48 and Carpenter, 2007; Tomasello and Moll, 2010). Series of experiments showed that

49 chimpanzees (Pan troglodytes) are equivalent to humans when it comes to cognitive skills for

50 dealing with the physical world (e.g., space, causal reasoning), but lag behind substantially in

51 the social realm (e.g., high-fidelity imitative learning, cooperative communication; Herrmann

52 et al. 2007; Tomasello and Moll 2010). In turn, it has been suggested that these reduced

53 socio-cultural capacities preclude chimpanzees from understanding the roles of joint activities

54 and sharing joint goals, the prerequisites for shared intentionality (see (Tomasello and Moll,

55 2010)).

Recent work, however, has challenged this view and proposed to seek for

57 evolutionary precursors of human shared intentionality not with experimental designs but 
58 within naturally occurring contexts of interacting animals (Genty et al., 2020; Heesen et al.,

59 2020, 2017). From an interactional perspective, correlates of shared intentionality could

60 manifest in the establishment of joint attention, joint commitment and joint actions, given

61 their requisite functions for sharing psychological states (Enfield and Levinson, 2006; Genty

62 et al., 2020). Bonobos, for example, have been observed to use communication to facilitate

63 re-engagement of the partner during social games (Pika and Zuberbühler, 2008), and

64 naturally occurring interactions (Heesen et al., 2020), which attests to their commitment to

65 joint activities (Genty et al., 2020). Given the interactional nature of human culture (Enfield

66 and Levinson, 2006; Tomasello, 2019) and the quest for what makes human culture unique

67 (Boyd et al., 2011; Ramsey, 2013; Richerson and Boyd, 2005), an important outstanding

68 question is whether great apes similarly display (correlates of) shared intentionality during

69 the execution of their joint cultural practices.

70 Here, we investigate whether chimpanzees communicate their desire to engage

71 partners in a joint cultural practice requiring the coordination of actions - the grooming

72 handclasp (McGrew and Tutin, 1978). The grooming handclasp (henceforth "GHC") is a

73 cultural social-grooming variant defined as a symmetrical postural configuration in which

74 two partners simultaneously extend one of their arms overhead and clasp each other's

75 extended hand at the palm, wrist or forearm, while grooming each other with the other arm

76 (McGrew and Tutin, 1978; Nakamura and Uehara, 2004; van Leeuwen et al., 2012). While

77 the cultural nature of GHC has been firmly established (McGrew et al., 2001; Nakamura,

78 2002; van Leeuwen et al., 2017, 2012; Wrangham et al., 2016), little is known about the ways

79 in which chimpanzees (or bonobos: (Fruth et al., 2006)) coordinate the execution of the GHC,

80 other than one individual (i.e., the initiator) physically shaping the body of the envisioned

81 partner into the GHC posture (De Waal and Seres, 1997). To learn more about this

82 coordination process, we studied the onset of GHCs with frame-by-frame analysis and 
83 compared the observed behaviors with matched-control windows (i.e., social grooming

84 events of the same partners without GHC) (sensu De Waal and Yoshihara 1983).

85 Furthermore, we examined whether chimpanzees initiated GHCs flexibly or in ritualized

86 sequences to shed light on their interactional versatility. Lastly, we inspected whether

87 chimpanzees showed signs of determination/goal-directedness to perform this joint action

88 together with their allocated partner, by identifying the use of persistence and/or elaboration

89 of initial initiation strategies when the desired response (i.e., the partner's commitment to

90 GHC) remained absent. In conjunction, these investigations may illuminate if great apes have

91 the capacity and motivation to engage in socio-cultural practices by means of (correlates of)

92 shared intentionality. 


\section{Results}

\section{GHC initiations}

95 We examined GHC initiations in a group of 52 semi-wild chimpanzees (Table S1). To

96 determine the mechanisms underlying GHC initiation, we investigated the occurrences of ten

97 selected chimpanzee behaviors (Table S2 and Table S3) in a comparison between $i$ ) GHC

98 initiations and initiations of regular grooming bouts, and ii) pre-handclasp (PH) periods and

99 their matched-control (MC) windows (see Methods and Figure 1). Four behaviors ("head

100 touch", "nosewipe", "self-scratch" and "torso") occurred both during GHC initiations and

101 initiations of regular grooming bouts (>15\% of the bouts; see Table S3), and were thus not

102 considered unique to GHC initiations. Six behaviors were observed infrequently during

103 initiations of regular grooming bouts $(<15 \%)$ and more frequently in the $\mathrm{PH}$ compared to the

104 MC context (Wilcoxon signed-rank: all $p<0.04$, Holm-corrected; $n_{\mathrm{ph}-\mathrm{mc}}=94 ; n_{\text {ind }}=33$; see

105 Table S4). These behaviors were thus considered specific to GHC initiations. Two of these

106 behaviors ("elbow hold" and "hand grab") corresponded to the practice of shaping (De Waal

107 and Seres 1997; e.g., Video S11), while the remaining behaviors ("elbow touch", "hand

108 touch", "head move", and "hold") did not involve any/prolonged physical contact with the

109 partner. These latter behaviors were considered to be potentially communicative, i.e., in the

110 form of gestures (Liebal and Call 2012; e.g., Video S12). 

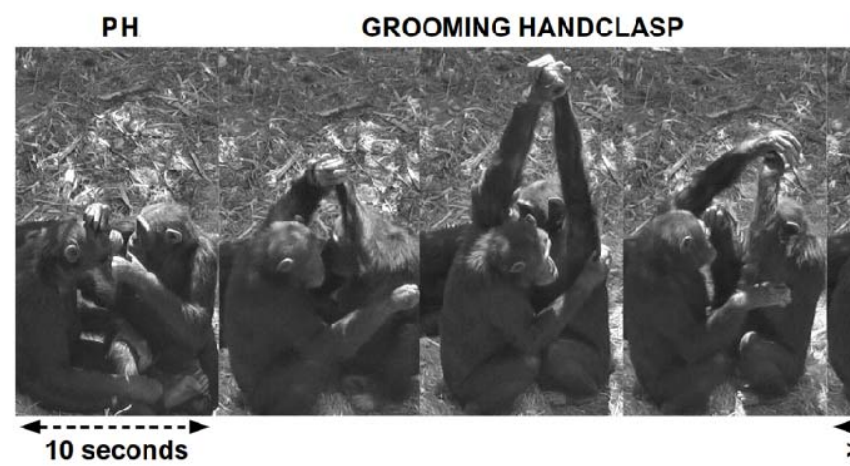

POST GHC

MC BOUT END

112 Figure 1. GHC bout including the identified pre-handclasp (PH) and matched-control period (MC).

113 The PHs and MCs were chosen to exactly match in terms of individuals, bodily positioning and 114 activities (grooming) in order to identify mechanisms specific to the initiation of GHC.

Gestures are defined as bodily actions that are mechanically ineffective and recipient-

117 directed, and result in a voluntary response from the recipient (Pika, 2008; Liebal and Call

118 2012). The four potentially communicative GHC-initiation behaviors followed this definition

119 - they were mechanically ineffective bodily actions resulting in voluntary GHC responses.

120 Only for "head move" we could envision partly non-intentional co-variation with other

121 behaviors like changing grooming posture or redirecting attention. "Elbow touch" and "hand

122 touch" involved targeted physical contact from actor to recipient and were thus recipient-

123 directed, and during "hold" and "head move" signalers faced their recipient in $100 \%$ of

124 observed instances ( $n=31$ and $n=18$, respectively). These gestures were produced flexibly,

125 with 14 of 15 individuals with more than one GHC initiation showing variation in the start

126 behavior (only counting the 6 GHC-specific behaviors determined above) of their initiation

127 sequences (Binomial test: $p<0.001$; also see Figure 2 and associated R-code). The gestures

128 were also produced in a goal-directed way, as indicated by the occurrence of elaboration (i.e.,

129 the use of additional behavior: Leavens et al., 2005) in $31 \%$ of the cases where an initial

130 gesture failed to initiate a GHC ( $n=10$ out of the 32 instances where gestures were used as an

131 initiation strategy, see Figure 2 and details below). Elaboration occurred after an average 
132 response waiting time of $0.5 \mathrm{~s}$ and took the form of another gesture $(n=4)$, a shaping behavior

$133(n=2)$, or a combination of both another gesture and a shaping behavior $(n=4)$ before the

134 GHC finally commenced. Taken together, these observations show that chimpanzees are

135 flexibly capable and determined to (re-)transmit their motivation to engage in GHC when

136 needed.

137 In general, of the $94 \mathrm{PH} / \mathrm{MC}$ comparison bouts, $21(22 \%)$ contained either or both

138 shaping behaviors ("elbow hold", "hand grab"), 32 (34\%) contained one or more of the four

139 communicative gestures ("elbow touch", "hand touch", "hold”, "head move") and no

140 shaping, and 41 (44\%) contained neither shaping nor potentially communicative behaviors.

141 We labeled the third type of GHC initiation as "synchronous", as the individuals appeared to

142 commit to the GHC near-simultaneously. Moreover, if any behavior was scored during the

143 PH window in the synchronous GHCs aside from the raising of the arms (which by definition

144 occurs before every GHC), these were behaviors common to the initiation of regular

145 grooming bouts (“head touch", "nosewipe", "self-scratch", "torso”, see Table S3). 


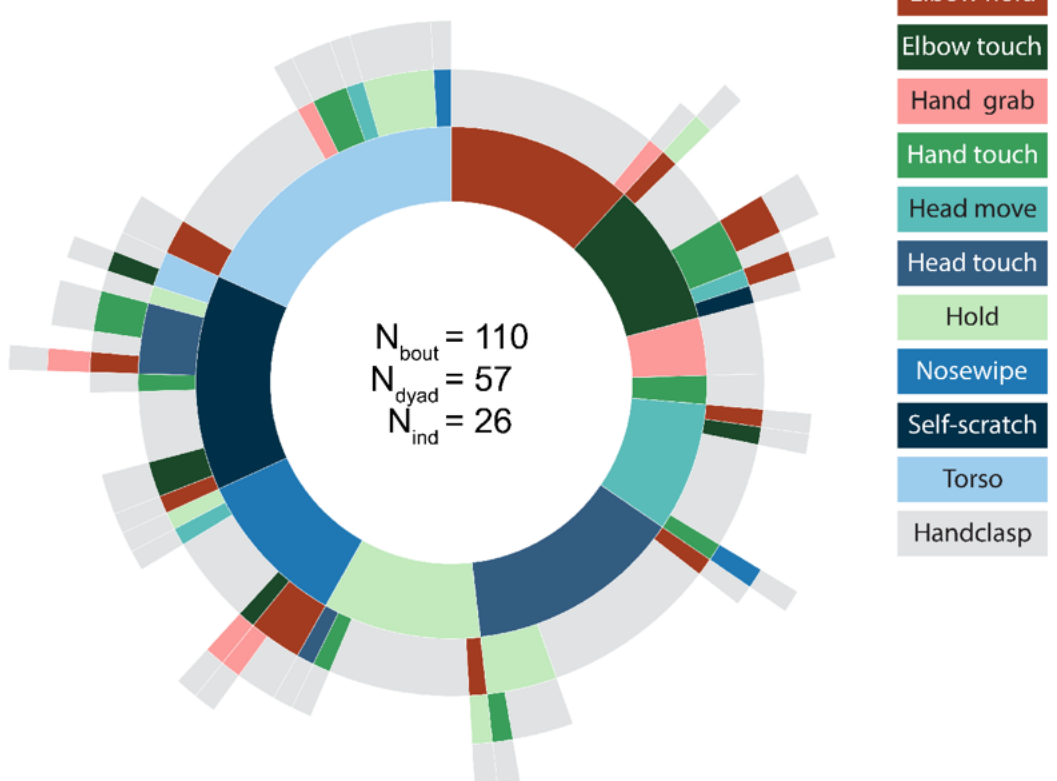

147 Figure $2 *$. Behavioral sequences by the initiator leading to GHC $(n=110)$. Starting behaviors are

148 depicted in the inner colored circle, with the grey outer circle being the endpoint of the sequence (i.e.,

149 GHC). In order to consider the full flexibility of all types of GHC initiations, we included the four

150 "synchrony" behaviors that are also common to the initiation of regular grooming bouts ("head

151 touch", "nosewipe", "self-scratch", and “torso"). * Interactive version available as Figure S13. 


\section{Discussion}

154 Our findings show that chimpanzees communicate to engage in one of their most enigmatic

155 socio-cultural practices, the grooming handclasp (McGrew and Tutin, 1978). To date, the

156 mechanism underlying the emergence and stability of the GHC culture in chimpanzees has

157 been described in terms of physical shaping (De Waal and Seres, 1997). By applying

158 matched-control methodologies (De Waal and Yoshihara, 1983) and following established

159 criteria in the field of communication (Leavens et al., 2005; Liebal and Call, 2012; Pika,

160 2008), we identify flexible and goal-directed gestural communication as an additional

161 mechanism by which chimpanzees initiate and coordinate their grooming handclasps.

162 The grooming handclasp is a cooperative activity that requires coordination for

163 successful execution. Chimpanzees cooperate (Mitani, 2009), but not much is known about

164 the ways in which they coordinate their joint efforts. In experimental settings, some

165 chimpanzees used location enhancing behaviors (e.g., bodily positioning, touching, peering)

166 (Melis and Tomasello, 2019), or generic gestures (e.g., arm fling, clapping, banging on

167 panels; Voinov et al. 2020) to entice their conspecific partners into a joint action. In natural

168 contexts, chimpanzees and bonobos communicate to coordinate joint actions like joint travel

169 (Fröhlich et al., 2016) and social play (Heesen et al., 2017; Hobaiter and Byrne, 2014), yet

170 “... the degree to which these actions are joint in terms of whether or not partners aim to

171 achieve shared goals together, or whether partners have shared intentions, remains unknown."

172 (Genty et al., 2020). Moreover, none of these examples pertain to cultural practices, while

173 joint and shared intentionality (Koreň, 2016; Tomasello and Carpenter, 2007) conducive to

174 joint action are so central to human accounts of culture (Enfield and Levinson, 2006;

175 Tomasello, 2019).

176 Our findings identify chimpanzees to use gestural communication in a natural context

177 to overcome a coordination challenge in the socio-cultural domain (McGrew and Tutin, 1978; 
178 Nakamura and Uehara, 2004; van Leeuwen et al., 2012). The socio-cultural interaction was

179 not just shaped by one invested individual (De Waal and Seres, 1997), but when the initiator

180 communicated its desire to engage in the GHC (e.g., by holding out its flexed arm at face

181 level in front of the desired partner), an active commitment in the form of a complementary

182 action by the partner was required to accomplish the interaction. As such, when

183 communicated, the cultural GHC practice appeared to ensue with a degree of joint

184 commitment regulating the coordination of the required actions, similar to the handshake

185 example in humans. We conclude that chimpanzees synchronize their attention to a joint

186 cultural action to which they are both committed, which suggests that chimpanzee social

187 culture, like human culture, may be founded on (correlates of) shared intentionality (cf.

188 (Tomasello et al., 2012; Tomasello and Carpenter, 2007; Tomasello and Moll, 2010)).

189 Reflecting on these findings, we posit that also for animals the socio-cultural context

190 i) facilitates and shapes mechanisms conducive to coordinating joint actions (also see (Genty

191 et al., 2020)), and ii) provides the adequate breeding ground for the transformation of

192 individualistic to collaborative predispositions and capacities - a transformation thought to be

193 unique to the evolution of the human species (Tomasello and Carpenter, 2007). Regarding $i$ ),

194 the socio-cultural property of the GHC (McGrew et al., 2001; Nakamura, 2002; van Leeuwen

195 et al., 2012) entails a group-level engagement which spurs in individuals both the motivation

196 to instigate and be susceptible to requests for interaction and, in the case of initiating GHC,

197 solve a coordination challenge. Whereas at first this context may produce overzealous

198 individuals who physically shape their naïve partners into GHC (De Waal and Seres, 1997),

199 in a later stage, the shared readiness to coordinate this joint cultural practice may catalyze the

200 transformation of one-sided shaping (only one actor) into two-sided communication (two

201 committed actors). This hypothesis would $a$ ) explain why communication for coordination

202 purposes has not been readily observed in chimpanzees (i.e., cultural contexts have been 
203 overlooked) (Duguid et al., 2020a, 2020b), and b) predict more communication and seamless

204 coordination in more experienced dyads as focus for future research. Regarding ii), it has

205 been posited that humans are unique in their cooperative engagement owing to their

206 capacities for shared intentionality (Tomasello, 2019). Humans are thought to transform

207 individualistic activities into joint, collaborative activities during ontogeny and especially

208 throughout socio-cultural immersion - a process presumably absent in chimpanzees

209 (Tomasello and Carpenter, 2007). It may thus be no coincidence that exactly in this socio-

210 cultural GHC-context we observed capacities for joint attention and commitment in

211 chimpanzees, similar to the ones ascribed to young children engaging in joint activities

212 (Tomasello, 2019). The ontogeny of chimpanzees' cultural engagement with a specific focus

213 on elements of shared intentionality may provide exciting insights for theories on the

214 evolution of human thinking (Heyes, 2018; Tomasello, 2019). 


\section{Methods}

\section{Subjects}

218 Subject were 52 chimpanzees (Table S1) at the Chimfunshi Wildlife Orphanage, Zambia.

219 GHC has been a customary behavior in the group for over 15 years, and 39 individuals

220 (including all 32 adults) were observed to GHC at least once. The study was approved by the

221 Chimfunshi Research Advisory Board (permit: CWOT_2019C039), and conformed to the

222 nationwide legal requirements. Chimfunshi is accredited by PASA and adheres to the rules

223 and regulations with respect to animal care and management as stipulated by the Zambia

224 Wildlife Authority.

\section{Collection \& Coding}

Data were collected by ZG from 23-03-2019 to 04-06-2019 between 8am-4pm with handheld

digital video cameras (Panasonic HDC-HS100). To capture GHC initiations, filming

commenced as soon as two individuals approached one another. Filming continued if the individuals started social grooming and lasted until they (a) had stopped grooming for over

23130 seconds, (b) started grooming another individual, or (c) physically separated. A grooming

232 bout was defined as running from the start of grooming until the moment one of the

233 aforementioned ending conditions was met. A bout was considered a GHC-bout if it

234 contained one or multiple GHCs, and a regular grooming bout if no GHCs occurred.

GHC-bouts had either a side or back view and were analyzed for initiation behaviors

if a $10 \mathrm{sec}$ pre-handclasp (PH) social grooming window was available before the first GHC in

237 the bout. We only analyzed the initiation of the first GHC in GHC-bouts, because previous

238 GHCs could possibly function as signals for subsequent GHCs. The start of a GHC was

239 defined as the instance of handclasp above face level; the end as the instance that physical 
240 contact of the palms/wrists was broken. Moreover, a Matched-Control (MC) period was

241 recorded to enable comparison of individual initiation behaviors across conditions (De Waal

242 and Yoshihara, 1983). The MC-period was defined as a 10sec-window minimally 10sec after

243 the last GHC occurrence in the GHC bout, in which the individuals had to be positioned in

244 the same relative positions as during the GHC, while still engaging in social grooming

245 (Figure 1). Additionally, initiations of regular, non-GHC-bouts were opportunistically

246 recorded ( $n_{\text {side and back }}=23$, Table S3) to identify behaviors used in the initiation of regular

247 grooming bouts.

248 Videos were scored in ELAN (Wittenburg et al., 2006), behaviors were coded based

249 on preliminary screening of the videos and established chimpanzee ethograms (Nishida et al.,

250 1999) (see Table S2 and SI videos). An individual was considered the initiator of a GHC bout

251 when they were either a) the first one to produce a GHC-specific initiation behavior or b) in

252 the absence of these behaviors, the first to raise their arm for the GHC. A subset of $20 \%$ of

253 the data was coded by two further observers to establish IRR. Mean dyadic agreement was

2540.833 for coding behaviors (range $0.81-0.89$ ), and 0.973 for identifying the initiating

255 individual (range 0.89-1; see SI for details).

257 Analyses

258 Analyses were done in R 3.6.1. Non-parametric statistics were applied, including Bonferroni-

259 Holm corrections for multiple testing (Holm, 1979). For the PH-MC comparison, we

260 analyzed those behaviors that $i$ ) occurred $\geq 5$ times in GHC-bouts, and $i i$ ) did not also

261 frequently (>15\% of bouts) occur during initiations of regular grooming bouts (see Table S3).

262 Given that social grooming occurred in both PH and MC windows by definition (see Figure 1

263 and "Collection \& Coding"), we did not consider the grooming behaviours (see Table S3) as 
264 possible signals for GHC initiation. We only used GHC-bouts with a side-view (optimal

265 vantage point) and a Matched Control period ( $n=94)$. Auxiliary analysis including also back-

266 view observations $\left(n_{\text {total }}=133\right.$ ) supported our main analyses (all 6 behaviors $p<0.02$, Holm

267 corrected; see Tables S3 \& S4). When assessing flexibility and elaboration in GHC initiations

268 (Figure 2), we used all side-view bouts regardless of the presence of MCs $(n=110)$.

\section{Data availability statement}

271 The code and data used for analyses are available via http://doi.org/10.5281/zenodo.4616274.

\section{Acknowledgments}

274 We are grateful to the Chimfunshi Wildlife Orphanage Trust for their ongoing support of our

275 research endeavors. In particular we wish to thank the staff of Chimfunshi, in particular

276 manager Innocent Mulenga, as well as Jake Brooker for his assistance during data collection

277 and inter-rater-reliability.

\section{Competing interests}

280 The authors declare to have no competing interests.

\section{Compliance with Ethical Standards}

283 The authors declare no conflict of interests to exist. The research on chimpanzees was strictly non-invasive/observational and adhered to the ethical guidelines provided by the Chimfunshi 
285 Research Advisory Board. All applicable international, national, and/or institutional

286 guidelines for the care and use of animals were followed.

287

\section{Funding}

289 EJCvL was funded by a Postdoctoral Fellowship (12W5318N) awarded by the Research

290 Foundation Flanders (FWO). ZG was supported by the ABS Student Grant from the Animal

291 Behavior Society. The authors declare no competing interests. 


\section{References}

294 Boyd R, Richerson PJ, Henrich J. 2011. The cultural niche: Why social learning is essential

295 for human adaptation. Proc Natl Acad Sci U S A 108:10918-10925.

296 doi:10.1073/Pnas.1100290108

De Waal FBM, Seres M. 1997. Propagation of handclasp grooming among captive

298 chimpanzees. Am J Primatol 43:339-346. doi:10.1002/1098-2345

De Waal FBM, Yoshihara D. 1983. Reconciliation and Redirected Affection in Rhesus Monkeys. Behaviour 85:224-241. doi:10.1163/156853983X00237

Duguid S, Allritz M, de las Heras A, Nolte S, Call J. 2020a. Cooperation and communication

302 in great apes In: Hopper LM, Ross SR, editors. Chimpanzees in Context. Chicago:

$303 \quad$ University of Chicago Press. pp. 305-329.

304 Duguid S, Wyman E, Grueneisen S, Tomasello M. 2020b. The Strategies Used by

305 Chimpanzees (Pan troglodytes) and Children (Homo sapiens) to Solve a Simple

306 Coordination Problem. J Comp Psychol 134:401-411. doi:10.1037/com0000220

307 Enfield NJ, Levinson SC. 2006. Roots of human sociality: culture, cognition and interaction.

$308 \quad$ Berg.

309 Fröhlich M, Kuchenbuch P, Müller G, Fruth B, Furuichi T, Wittig RM, Pika S. 2016.

310 Unpeeling the layers of language: Bonobos and chimpanzees engage in cooperative

311 turn-taking sequences. Sci Rep 6:25887. doi:10.1038/srep25887

312 Fruth BI, Hohmann G, Beuerlein MM, Mcgrew WC. 2006. Grooming Hand Clasp by

313 Bonobos of Lui Kotale, Democratic Republic of Congo. Pan Africa News 13:6-8.

314 Genty E, Heesen R, Guery J-P, Rossano F, Zuberbühler K, Bangerter A. 2020. How apes get

315 into and out of joint actions: Shared intentionality as an interactional achievement. 
317 Heesen R, Bangerter A, Zuberbühler K, Rossano F, Iglesias K, Guéry J-P, Genty E. 2020.

318 Bonobos engage in joint commitment. Sci Adv 6:eabd1306. doi:10.1126/sciadv.abd1306

319 Heesen R, Genty E, Rossano F, Zuberbühler K, Bangerter A. 2017. Social play as joint

320 action: A framework to study the evolution of shared intentionality as an interactional

321 achievement. Learn Behav 45:390-405. doi:10.3758/s13420-017-0287-9

322 Henrich J. 2016. The Secret Of Our Success: How Culture Is Driving Human Evolution,

323 Domesticating Our Species, and Making Us Smarter. Princeton University Press.

324 Herrmann E, Call J, Hernandez-Lloreda M V, Hare B, Tomasello M. 2007. Humans have

325 evolved specialized skills of social cognition: The cultural intelligence hypothesis.

$326 \quad$ Science (80- ) 317:1360-1366. doi:Doi 10.1126/Science.1146282

327 Heyes CM. 2018. Cognitive gadgets $\square$ : the cultural evolution of thinking. Belknap Press.

328 Hobaiter C, Byrne RW. 2014. The Meanings of Chimpanzee Gestures. Curr Biol 24:1596-

329 1600. doi:10.1016/J.CUB.2014.05.066

330 Holm S. 1979. A Simple Sequentially Rejective Multiple Test Procedure. Scand J Stat 6:65-

$331 \quad 70$.

332 Koreň L. 2016. Joint intentionality: From thin to thick. J Soc Ontol 2:75-85. doi:10.1515/jso2015-0047

Laland KN, Janik VM. 2006. The animal cultures debate. Trends Ecol Evol 21:542-547. doi:Doi 10.1016/J.Tree.2006.06.005

336 Leavens DA, Russell JL, Hopkins WD. 2005. Intentionality as measured in the persistence and elaboration of communication by chimpanzees (Pan troglodytes). Child Dev 
76:291-306. doi:10.1111/j.1467-8624.2005.00845.x

339 Liebal K, Call J. 2012. The origins of non-human primates' manual gestures. Philos Trans $R$

$340 \quad$ Soc Lond B Biol Sci 367:118-28. doi:10.1098/rstb.2011.0044

341 McGrew WC, Marchant LF, Scott SE, Tutin CEG. 2001. Intergroup differences in a social

342 custom of wild chimpanzees: The grooming hand-clasp of the Mahale Mountains. Curr

$343 \quad$ Anthropol 42:148-153.

344 McGrew WC, Tutin CEG. 1978. Evidence for a Social Custom in Wild Chimpanzees? Man

$345 \quad$ 13:234-251.

346 Melis AP, Tomasello M. 2019. Chimpanzees (Pan troglodytes) coordinate by communicating

347 in a collaborative problem-solving task. Proc R Soc B Biol Sci 286:20190408.

348 doi:10.1098/rspb.2019.0408

349 Mitani JC. 2009. Cooperation and competition in chimpanzees: Current understanding and

350 future challenges. Evol Anthropol 18:215-227. doi:10.1002/evan.20229

351 Nakamura M. 2002. Grooming-hand-clasp in Mahale M Group chimpanzees: implications for

352 culture in social behaviours In: Boesch C, Hohmann G, Marchant LF, editors.

353 Behavioural Diversity in Chimpanzees and Bonobos. Cambridge: Cambridge University

$354 \quad$ Press. pp. 71-83.

355 Nakamura M, Uehara S. 2004. Proximate factors of different types of grooming hand-clasp in

356 mahale chimpanzees: Implications for chimpanzee social customs. Curr Anthropol

$357 \quad 45: 108-114$.

358 Nishida T, Kano T, Goodall J, McGrew WC, Nakamura M. 1999. Ethogram and

359 Ethnography of Mahale Chimpanzees. Anthropol Sci 107:141-188.

$360 \quad$ doi:10.1537/ase. 107.141 
361 Oxlund B. 2020. An Anthropology of the Handshake. Anthropol Now 12:39-44.

362 doi:10.1080/19428200.2020.1761216

363 Pika S. 2008. Gestures of apes and pre-linguistic human children: Similar or different? First

$364 \quad$ Lang 28:116-140. doi:10.1177/0142723707080966

365 Pika S, Zuberbühler K. 2008. Social games between bonobos and humans: Evidence for

366 shared intentionality?, American Journal of Primatology. doi:10.1002/ajp.20469

367 Ramsey G. 2013. Culture in humans and other animals. Biol Philos 28:457-479.

368 doi:10.1007/s10539-012-9347-x

369 Richerson PJ, Boyd R. 2005. Not by genes alone: how culture transformed human evolution.

$370 \quad$ Chicago: The University of Chicago Press.

371 Tomasello M. 2019. Becoming human: A theory of ontogeny. Cambridge, MA.: Harvard

$372 \quad$ University Press.

373 Tomasello M, Carpenter M. 2007. Shared intentionality. Dev Sci 10:121-125.

374 doi:10.1111/j.1467-7687.2007.00573.x

375 Tomasello M, Melis AP, Tennie C, Wyman E, Herrmann E. 2012. Two key steps in the

376 evolution of human cooperation. Curr Anthropol 53:673-692. doi:10.1086/668207

377 Tomasello M, Moll H. 2010. The gap is social: Human shared intentionality and culture In:

378 Kappeler PM, Silk JB, editors. Mind the Gap: Tracing the Origins of Human Universals.

379 Berlin: Springer. pp. 331-349. doi:10.1007/978

380 van Leeuwen EJC, Cronin KA, Haun DBM, Mundry R, Bodamer MD. 2012. Neighbouring

381 chimpanzee communities show different preferences in social grooming behaviour. Proc

$382 \quad$ Biol Sci 279:4362-7. 
383 van Leeuwen EJC, Mundry R, Cronin KA, Bodamer M, Haun DBM. 2017. Chimpanzee

384 culture extends beyond matrilineal family units. Curr Biol 27:588-590.

$385 \quad$ doi:10.1016/j.cub.2017.05.003

386 Voinov P V., Call J, Knoblich G, Oshkina M, Allritz M. 2020. Chimpanzee Coordination and

387 Potential Communication in a Two-touchscreen Turn-taking Game. Sci Rep 10:3400.

$388 \quad$ doi:10.1038/s41598-020-60307-9

389 Wittenburg P, Brugman H, Russel A, Klassmann A, Sloetjes H. 2006. ELAN: a Professional

390 Framework for Multimodality Research Proceedings of the 5th International Conference

391 on Language Resources and Evaluation (LREC).

392 Wrangham RW, Koops K, Machanda ZP, Worthington S, Bernard AB, Brazeau NF,

393 Donovan R, Rosen J, Wilke C, Otali E, Muller MN. 2016. Distribution of a Chimpanzee

394 Social Custom Is Explained by Matrilineal Relationship Rather Than Conformity. Curr

395 Biol 26:3033-3037. doi:10.1016/j.cub.2016.09.005 


\section{Supplementary Information captions}

399 SI_1. Supplementary information and tables, including subject demographics, the behavioral

400 ethogram, frequency table of behavior, outcomes of the Wilcoxon-signed rank tests,

401 and details on the inter-rater reliability calculation.

402 Video S1. Elbow hold.

403 Video S2. Elbow touch.

404 Video S3. Hand grab.

405 Video S4. Hand touch.

406 Video S5. Head move.

407 Video S6. Head touch.

408 Video S7. Hold.

409 Video S8. Nosewipe.

410 Video S9. Self-scratch.

411 Video S10. Torso.

412 Video S11. Video example of a grooming handclasp initiated with shaping behavior.

413 Video S12. Video example of a grooming handclasp initiated with gestural communication.

414 Interactive Figure S13. Interactive version of Figure 2. 


\section{Supplementary Information}

\section{Chimpanzees communicate to coordinate a cultural practice}

417 Zoë Goldsborough ${ }^{1,2}$, Anne Marijke Schel, Edwin J. C. van Leeuwen*

$418{ }^{1}$ Animal Ecology, Department of Biology, Utrecht University, Utrecht, the Netherlands

$419{ }^{2}$ Department for the Ecology of Animal Societies, Max Planck of Animal Behavior,

420 Konstanz, Germany

421 *Corresponding author: ejcvanleeuwen@gmail.com

Table S1. Subject demography of Group 2 at Chimfunshi Wildlife Orphanage on 01-072019.

427

428

429

430

\begin{tabular}{l|lll}
\cline { 2 - 4 } & Male & Female & Total \\
\hline Adult (> 12 years) & 8 & 24 & 32 \\
Juvenile (3-11 years) & 7 & 5 & 12 \\
Infant (< 3 years) & 3 & 5 & 8 \\
\hline
\end{tabular}

Table S2. Ethogram of behavior, with reference to supplementary videos of behaviors.

\begin{tabular}{|c|c|c|}
\hline Behavior & Video & Definition \\
\hline Elbow Hold & S1 & $\begin{array}{l}\text { Places own hand on elbow or arm of other, maintaining physical } \\
\text { contact as arm of other moves }\end{array}$ \\
\hline Elbow Touch & S2 & Briefly touches elbow or arm of other with own hand \\
\hline Hand Grab & S3 & $\begin{array}{l}\text { Grabs hand of other with own hand, maintaining contact as arm or } \\
\text { hand of other moves }\end{array}$ \\
\hline Hand Touch & $\mathrm{S} 4$ & Briefly touches hand of other with own hand \\
\hline Head Move & S5 & Tilts head up or downwards \\
\hline Head Touch & S6 & Touches (side of) head of other with hand, brief or prolonged contact \\
\hline Hold & S7 & $\begin{array}{l}\text { Holds arm up in the air at peak of arm-raise movement, i.e., the raising } \\
\text { of the upper arm with some flexion in the elbow }\end{array}$ \\
\hline Nosewipe & S8 & Swipes hand across or underneath nose in quick motion \\
\hline Self-scratch & S9 & Drags hand across body in long rough strokes \\
\hline Torso & S10 & Turns torso towards or away from other \\
\hline
\end{tabular}


434 Table S3. Occurrence of all ethogram behaviors in pre-handclasp (PH) and matched-control 435 (MC) periods, as well as initiation of regular grooming bouts (IRG). Reported is the total 436 number of instances and between brackets the number of instances divided by the number of 437 bouts.

438

\begin{tabular}{lllllll}
\hline & \multicolumn{3}{c}{ Side view } & \multicolumn{3}{c}{ Side and back view } \\
\hline Behavior & PH $(\mathbf{n}=$ & $\mathbf{M C}(\mathbf{n}=$ & IRG $(\mathbf{n}=$ & $\mathbf{P H}(\mathbf{n}=$ & $\mathbf{M C}(\mathbf{n}=$ & IRG $(\mathbf{n}=$ \\
& $\mathbf{9 4})$ & $\mathbf{9 4})$ & $\mathbf{1 7})$ & $\mathbf{1 3 3})$ & $\mathbf{1 3 3})$ & $\mathbf{2 3})$ \\
\hline Elbow hold & $18(0.19)$ & $1(0.01)$ & $0(0)$ & $25(0.19)$ & $1(0.01)$ & $0(0)$ \\
Elbow touch & $14(0.15)$ & $0(0)$ & $0(0)$ & $16(0.12)$ & $0(0)$ & $0(0)$ \\
Groom face & $29(0.31)$ & $44(0.47)$ & $7(0.41)$ & $41(0.31)$ & $63(0.47)$ & $8(0.35)$ \\
Groom & $24(0.26)$ & $25(0.27)$ & $5(0.29)$ & $28(0.21)$ & $32(0.24)$ & $6(0.26)$ \\
hand/arm & & & & & & \\
Groom other & $53(0.56)$ & $70(0.74)$ & $8(0.47)$ & $79(0.59)$ & $95(0.71)$ & $14(0.61)$ \\
Hand grab & $5(0.05)$ & $0(0)$ & $0(0)$ & $8(0.06)$ & $0(0)$ & $0(0)$ \\
Hand touch & $6(0.06)$ & $0(0)$ & $0(0)$ & $6(0.05)$ & $0(0)$ & $0(0)$ \\
Head move & $11(0.12)$ & $1(0.01)$ & $0(0)$ & $17(0.12)$ & $1(0.01)$ & $1(0.04)$ \\
Head touch & $14(0.15)$ & $3(0.03)$ & $3(0.18)$ & $18(0.14)$ & $3(0.03)$ & $4(0.17)$ \\
Hold & $14(0.15)$ & $1(0.01)$ & $1(0.06)$ & $16(0.12)$ & $1(0.01)$ & $1(0.04)$ \\
Nosewipe & $12(0.13)$ & $2(0.02)$ & $6(0.35)$ & $16(0.12)$ & $2(0.02)$ & $7(0.30)$ \\
Self-scratch & $25(0.27)$ & $9(0.10)$ & $3(0.18)$ & $31(0.23)$ & $13(0.10)$ & $5(0.22)$ \\
Torso & $30(0.32)$ & $0(0)$ & $10(0.59)$ & $44(0.33)$ & $0(0)$ & $11(0.48)$ \\
\hline
\end{tabular}

440

Table S4. Results of the paired Wilcoxon signed rank test of behavior frequency between the $442 \mathrm{PH}$ and MC periods.

443

\begin{tabular}{lllllll}
\hline & \multicolumn{3}{c}{ Side view $(\mathbf{n}=94)$} & \multicolumn{2}{c}{ Side and back view $(\mathbf{n}=133)$} \\
\hline Behavior & $\mathbf{V}$ & $\begin{array}{l}\text { P-value } \\
(\text { raw })\end{array}$ & $\begin{array}{l}\text { P -value (adjusted } \\
\text { Holm) }\end{array}$ & $\mathbf{V}$ & $\begin{array}{l}\text { P-value } \\
(\text { raw })\end{array}$ & $\begin{array}{l}\text { P -value (adjusted } \\
\text { Holm) }\end{array}$ \\
\hline Elbow hold** & 10 & $<0.001$ & 0.001 & 13.5 & $<0.001$ & $<0.001$ \\
Elbow touch* & 0 & $<0.001$ & 0.001 & 0 & $<0.001$ & $<0.001$ \\
Hand grab* $^{*}$ & 0 & 0.037 & 0.039 & 0 & 0.006 & 0.012 \\
Hand touch* & 0 & 0.020 & 0.039 & 0 & 0.020 & 0.020 \\
Head move** & 6.5 & 0.004 & 0.013 & 8.5 & $<0.001$ & 0.001 \\
Hold** & 0 & $<0.001$ & 0.001 & 0 & $<0.001$ & $<0.001$ \\
\hline
\end{tabular}


448 To establish inter-rater reliability, two additional coders (JB and AM) analyzed the PHs and

449 MCs of $20 \%(\mathrm{~N}=19)$ of the GHC bouts. Additionally, ZG coded this subset for a second

450 time to compare to her first coding one year prior.

451 Due to the continuous nature of the behavioral scoring and the importance of

452 observers not only agreeing on the presence of (often rare) behaviors but also the order in

453 which they occurred, the usual Cohen's Kappa approach was not feasible. We calculated

454 inter-rater reliability as follows (see also attached data for each rater's coding and an

455 overview):

456 If raters both scored the same behavior in the same order at the same time (within 1

457 second) it was noted as an "agree", if either of the raters scored a behavior the other one did

458 not score it was a "disagree". As an informed estimation for the total amount of behaviors per

459 video we used the sum of the instances of agree and disagree. To correct for the probability of

460 two raters agreeing on a behavior by chance, we multiplied the probability of scoring a

461 particular behavior (1/11, the amount of different behaviors) by the probability of scoring it

462 for a certain individual (0.5).

463 The final calculation of the reliability score combined this probability of agreement

464 based on chance (POA) with the relative observer agreement (ROA, defined as agreed

465 behaviors/total amount of behaviors), in the form of $\frac{R O A-P O A}{1-P O A}$. For instance, 50 agrees out of

46670 total behaviors leads to a reliability score of $\frac{\left(\frac{50}{70}\right)-\left(0.5 * \frac{1}{11}\right)}{1-\left(0.5 * \frac{1}{11}\right)}=0.70$. For this study, all dyads

467 showed inter-rater reliability scores above $0.80\left(Z G_{-} 2-Z G_{-} 1=0.89 ;\right.$ JB-ZG_1 $=0.81$; AM-

468 ZG_1 $=0.82 ; \mathrm{JB}-\mathrm{AM}=0.81)$. Furthermore, each rater also coded who they thought initiated

469 the bout, and inter-rater reliability on initiator was calculated in a similar manner (with POA

470 being 0.5$)$ and was above 0.90 for all raters $\left(\mathrm{JB}-Z \mathrm{ZG}_{-} 1=0.89\right.$; all other dyads $\left.=1\right)$. 